\title{
Encoding via conjugate symmetries of slow oscillations for globally coupled oscillators
}

\author{
Peter Ashwin and Jon Borresen \\ Department of Mathematics, University of Exeter, Exeter EX4 4QE, United Kingdom
}

(Received 10 December 2003; published 10 August 2004)

\begin{abstract}
We study properties of the dynamics underlying slow cluster oscillations in two systems of five globally coupled oscillators. These slow oscillations are due to the appearance of structurally stable heteroclinic connections between cluster states in the noise-free dynamics. In the presence of low levels of noise they give rise to long periods of residence near cluster states interspersed with sudden transitions between them. Moreover, these transitions may occur between cluster states of the same symmetry, or between cluster states with conjugate symmetries given by some rearrangement of the oscillators. We consider the system of coupled phase oscillators studied by Hansel et al. [Phys. Rev. E 48, 3470 (1993)] in which one can observe slow, noise-driven oscillations that occur between two families of two cluster periodic states; in the noise-free case there is a robust attracting heteroclinic cycle connecting these families. The two families consist of symmetric images of two inequivalent periodic orbits that have the same symmetry. For $N=5$ oscillators, one of the periodic orbits has one unstable direction and the other has two unstable directions. Examining the behavior on the unstable manifold for the two unstable directions, we observe that the dimensionality of the manifold can give rise to switching between conjugate symmetry orbits. By applying small perturbations to the system we can easily steer it between a number of different marginally stable attractors. Finally, we show that similar behavior occurs in a system of phase-energy oscillators that are a natural extension of the phase model to two dimensional oscillators. We suggest that switching between conjugate symmetries is a very efficient method of encoding information into a globally coupled system of oscillators and may therefore be a good and simple model for the neural encoding of information.
\end{abstract}

DOI: 10.1103/PhysRevE.70.026203

PACS number(s): 05.45.Xt

\section{INTRODUCTION}

Much attention has been paid to the dynamics of systems of coupled nonlinear oscillators, with motivation from many disciplines including neural network computing [1] and theoretical neurosciences [2-4]. Among the class of possible coupled systems, globally coupled systems are particularly useful in gaining an understanding of dynamical effects such as clustering, even though realistic models are usually not of this form. Globally coupled systems show a variety of effects that are expected in highly connected systems (such as the glomeruli in the antennal lobes of insects [5], or see Ref. [6] for a general discussion of coupled systems). Of particular interest are cluster states where the oscillators split into a number of groups each of which is synchronized within itself but so that oscillators in different groups are not synchronized.

Previous investigations into cluster states of globally coupled systems include exploring the effect of varying coupling strengths on bifurcation of cluster states [7] and the effect of differing network topologies [8]. This has given a good understanding of cluster dynamics, especially for (attracting) equilibrium or periodic states. However, even if such periodic cluster states are not attractors, they may appear within attractors for globally coupled systems due to the effects of "slow switching" [9-11]. This is a phenomenon characterized by the appearance of robust attracting heteroclinic cycles within the dynamics. Similar switching behavior is found in systems coupled by delayed pulses [11] with the difference that they can give rise to "unstable attractors" [12].

Many mathematical models are available to describe the collective behavior of individual neurons $[13,14]$ and much effort has gone into modeling the action potential of spiking neurons through various excitatory and inhibitory coupling regimes. In the weak coupling limit, phase models can be obtained (see, for example, Ref. [15]). The possibility of robust heteroclinic cycles in such models was argued in Ref. [16] and confirmed by Hansel et al. [9,17] for an explicit system derived from Hodgkin-Huxley neurons with global weak coupling, where it was termed a "slow oscillation," and similar heteroclinic oscillations have been suggested by others as a mechanism for storage and retrieval of memory, for example, in the olfactory system $[2,18]$.

In this paper we return to the Hansel phase oscillator model in Sec. II and examine its dynamical behavior for $N$ $=5$ in detail. Section II focuses on analyzing the robust attracting heteroclinic behavior between periodic cluster states of the form $(a, a, a, b, b)$ (we describe the symmetries as $\mathbf{S}_{2} \times \mathbf{S}_{3}$ ). In particular we examine the geometry of the unstable manifolds and show in Sec. III that perturbations and noise can induce switching between conjugate symmetries. We suggest that the switching behavior is of considerable interest as a very simple model with a highly controllable and fast switching between a large number of simultaneously (but marginally) stable attractors. In particular, we show that one can move between any of the ten different conjugate versions of $\mathbf{S}_{2} \times \mathbf{S}_{3}$ using at most two switches.

To demonstrate that the dynamics we consider is not dependent on the reduction to a phase oscillator, we include here an investigation into a phase-energy model of Ashwin and Dangelmayr [19] that is a natural generalization from models of weakly dissipative Hamiltonian oscillators. By introducing a separate amplitude variable to mean field 
coupled phase oscillators, a phase-energy model with analogous coupling to that in Ref. [9] gives rise to the same behavior. We verify in Sec. IV that the switching effect also occurs in the latter system; we also discuss possible implications of this behavior.

\section{Dynamical clustering: A symmetry viewpoint}

We can view the dynamics of the globally coupled systems in terms of their symmetries, and use notation as in Ref. [16]. An all-to-all or globally coupled system of $N$ identical oscillators has symmetry groups corresponding to $\mathbf{S}_{N}$, the group of all permutations of $N$ objects. This corresponds to the system being equivalent under interchange of identical oscillators. Of course, it is not necessarily the case that the output from each oscillator will be identical. An $\mathbf{S}_{N}$ system of coupled nonlinear equations can support a wide range of dynamical behaviors depending on initial condition (e.g., Ref. [20]) and we are most interested in understanding the symmetries and dynamical structure of attractors for the system, i.e., the asymptotic dynamics of the systems for "typical" initial conditions.

Of particular interest in a globally coupled system is the appearance of partial synchronization or clustering. For a clustered state the output of the system of oscillators splits into several subgroups with asymptotically similar dynamics $[8,21]$. For example, a system of $N$ coupled oscillators, synchronized into to two clusters of size $m$ and $N-m$ the symmetry group of the dynamics is $\mathbf{S}_{m} \times \mathbf{S}_{N-m}$. The transition from a higher order synchronized state to a lower order (spontaneous symmetry breaking) is a common feature of coupled and symmetric systems.

\section{HANSEL, MATO, AND MEUNIER PHASE OSCILLATOR MODEL}

Hansel et al. [9] introduced a model for phase oscillators with the following governing equations, for $N$ oscillators with phases $\theta_{i} \in \mathbf{T}$ where $\mathbf{T}$ is the "one-torus" parametrized by $[0,2 \pi)$ and $i=1, \ldots, N$.

$$
\dot{\theta}_{i}=\omega+\frac{1}{N} \sum_{j=1}^{N} g\left(\theta_{i}-\theta_{j}\right)+\eta w_{i}(t) .
$$

The coupling function is chosen to include more than one harmonic,

$$
g(\phi)=-\sin (\phi+\alpha)+r \sin 2 \phi,
$$

where we note that the presence of both phase shifted and second harmonic terms represents a nonlinear mixture of stimulation and inhibition. In Ref. [9] this choice of $g(\phi)$ is motivated by a phase reduction of weakly coupled neural oscillators. The $w_{i}(t)$ are derivatives of uncorrelated white noise processes with unit variance per unit time and $\omega, \eta, \alpha$, and $r$ are real constants.

The system (1) clearly has $\mathbf{S}_{N}$ symmetry; it has an additional symmetry typical for weakly coupled oscillators, namely phase-translation symmetry,

$$
\theta_{i} \mapsto \theta_{i}+\rho,
$$

for any $\rho \in \mathbf{T}$, and so the system has symmetry $\mathbf{S}_{N} \times \mathbf{T}$. The phase-translation symmetry arises as a result of an averaging reduction and results in a major simplification in that periodic orbits of the systems can be reduced to relative equilibria of the action of $\mathbf{T}$; it was exploited in Ref. [16] to show that certain cluster states are generically present for $N$ coupled oscillators.

The model (1) has been studied by several authors including Kori and Kuramoto [10]. It provides an interesting example of a system of phase oscillators with an attracting robust heteroclinic cycle (a slow switching state) between $\mathbf{S}_{k} \times \mathbf{S}_{N-k}$ cluster states for $N$ oscillators. As discussed in Ref. [10] one can find attracting states that consist of heteroclinic cycles between a pair of two cluster states with division of the oscillators into groups of size $p N$ and $(1-p) N$ (these are between identical states if $p=\frac{1}{2}$ ). As in Ref. [9] we will consider an order parameter that gives a measure of the total coherence of the network

$$
\chi=\frac{1}{N}\left|\sum_{k=1}^{N} e^{i \theta_{k}}\right|
$$

The heteroclinic cycle manifests itself as a slow periodic oscillation of $\chi$, where the period of oscillation is roughly proportional to the reciprocal of the $\log$ of $\eta$. Observe that $\chi=1$ corresponds to synchronous oscillation, but for any other state $0 \leqslant \chi<1$.

For the system with $N=5$ oscillators the solutions with $\mathbf{S}_{2} \times \mathbf{S}_{3}$ clustering have ${ }^{5} C_{3}=10$ conjugate symmetries of the same type. This corresponds to permuting the entries in each of the symmetries. For example, if there is a periodic cluster state of the form $(a, a, a, b, b)$ then there will also be states $(a, b, b, a, a),(a, a, b, b, a)$, etc., corresponding to conjugate symmetries.

\section{Heteroclinic network for $N=5$ oscillators}

For parameters $\omega=1, r=0.25, \alpha=1.25, \eta=10^{-5}$, and $N$ $=5$ in system (1) we obtain the slow switching observed by Ref. [9] as in Fig. 1 (using a Runge-Kutta integrator with stepsize 0.1). The dynamics appear to be attracted to a robust heteroclinic cycle for all but a zero measure set of initial conditions. There is a heteroclinic cycle between two families of equilibria with symmetry $\mathbf{S}_{2} \times \mathbf{S}_{3}$. Schematically, this cycle is shown in Fig. 2. The asymptotic dynamics of this system, as noted by Refs. $[9,10]$ involves a division of the 


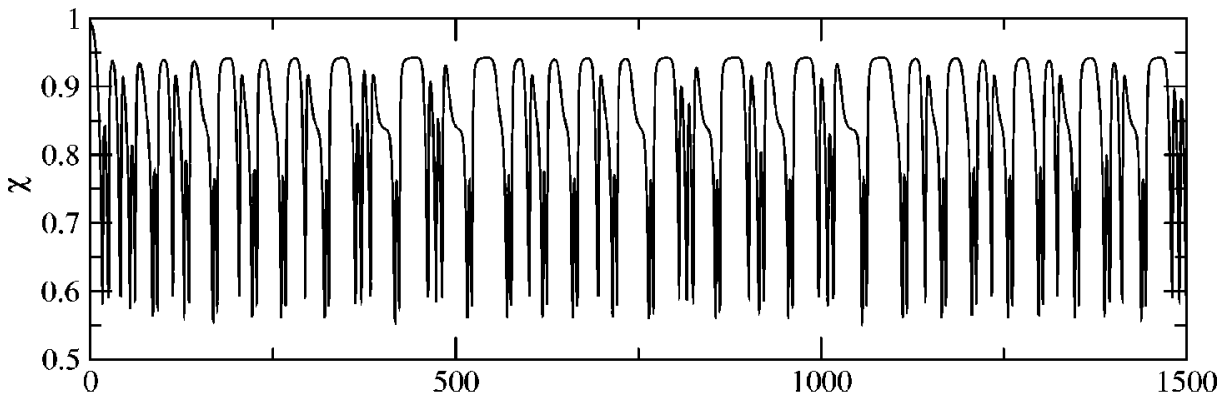

(a)

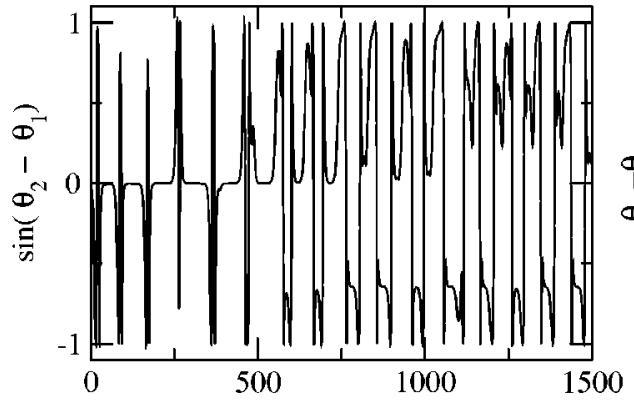

(b)

$\mathrm{t}$ $\mathbf{t}$

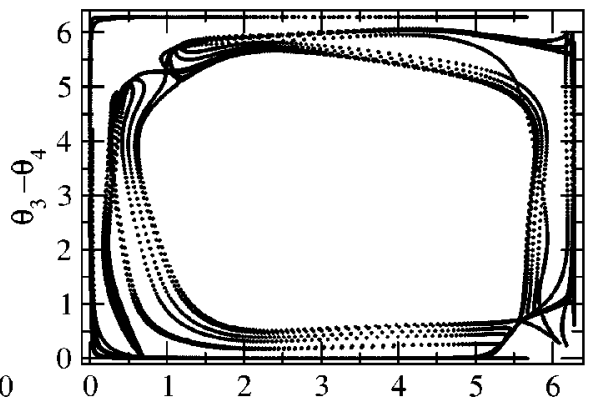

FIG. 1. (a)-(c) Time series plots of a slow switching state of five globally coupled phase oscillators. The presence of a small amount of noise means that it continues to cycle between two unstable periodic cluster states. Subplot (a) shows the order parameter $\chi$, (b) shows $\sin \left(\theta_{2}-\theta_{1}\right)$, while (c) shows two phase differences plotted against each other. Observe that (b) shows that the synchronization of $\theta_{1}$ and $\theta_{2}$ near the periodic clusters is lost near $t=500$. This is not evident in plot (a) of the order parameter. Note that in this and subsequent figures the units are dimensionless. oscillators into two clusters of size $p N$ and $(1-p N)$; in the case of $N=5$ this means that we can have clusters of the form $\mathbf{S}_{2} \times \mathbf{S}_{3}$ or $\mathbf{S}_{1} \times \mathbf{S}_{4}$. Only the former are involved in the global attractor, and in the absence of noise, the system performs ever more occasional transitions between just two periodic cluster states with $\mathbf{S}_{2} \times \mathbf{S}_{3}$ symmetry; we denote these states $a a a b b$ and $c c c d d$.

One of the main contributions of this paper is to note that the geometry of the connections between the invariant subspaces is much more complex than Fig. 2 would suggest. This is because $a a a b b$ has two unstable directions and $c c c d d$ has one unstable direction; hence the unstable manifold (after including points related by the $\mathbf{T}$ symmetry) for $a a a b b$ is two dimensional and incorporates an infinite number of trajectories. Of these trajectories, almost all of them converge to $c c c d d$, but a finite number of them converge elsewhere namely in this case to $c d d c c$, $d d c c c$, and $d c d c c$; we verify

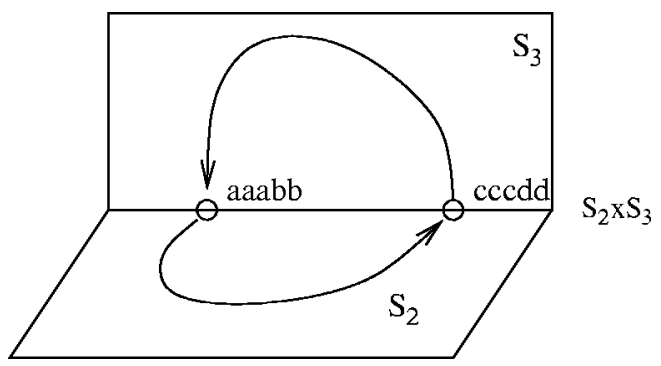

FIG. 2. Schematic showing the heteroclinic connections between the two saddle periodic orbits $a a a b b$ and $c c c d d$ within the invariant subspace $\mathbf{S}_{2} \times \mathbf{S}_{3}$. The periodic orbit $a a a b b$ has two unstable directions and corresponds to a breaking of the cluster of three oscillators. The orbit $c c c d d$ has one unstable direction corresponding to breaking the two cluster. Not shown are the invariant subspaces with symmetry $\mathbf{S}_{1} \times \mathbf{S}_{2} \times \mathbf{S}_{2}$ within $\mathbf{S}_{2}$; there are connections from $a a a b b$ to conjugate symmetry cluster states within these invariant subspaces. this by numerical simulations of a number of trajectories starting near $a a a b b$ within the subspace $(x, y, z, w, w)$ of points with symmetry $\mathbf{S}_{2}$, as shown in Fig. 3. This leads to possibility of noise-induced switching to slow oscillation to different but conjugate symmetries; we investigate this in the next section.

\section{SWITCHING BETWEEN CONJUGATE ATTRACTORS}

By considering the behavior of trajectories starting at one of the periodic orbits and exploiting symmetries one can map out the structure of the heteroclinic network. This amounts to an examination of trajectories such as those shown in Fig. 3. A schematic of the behavior on the unstable manifold of $a a a b b$ is shown in Fig. 4; this shows that there are connections from $a a a b b$ to $c d d c c, d c d c c$, and $d d c c c$ even though almost all trajectories pass to $c c c d d$. We also illustrate that the periodic orbits abbaa, babaa, and bbaaa are present in the closure of the unstable manifold even though they are not the asymptotic limit of any point in the unstable manifold. The unstable manifold of the orbits $c c c d d$, etc., are one dimensional (accounting for the $\mathbf{T}$ orbit) and only connections to the orbits $c c c b b$ with precisely the same symmetry.

Figure 5 shows the possible connections between periodic orbits $a a a b b$ and conjugate copies thereof. The ten conjugate orbits can be attained by choice of unstable manifold at the conjugate version of the periodic orbit $a a a b b$.

For the noise-free case we conjecture that typical trajectories will generically avoid the one-dimensional subset of connections in the unstable manifolds of the $a a a b b$. This is because in the absence of noise we have not observed switching between conjugate symmetries along trajectories that approach the heteroclinic network. Instead, we see a number of transitions always between the same symmetry 


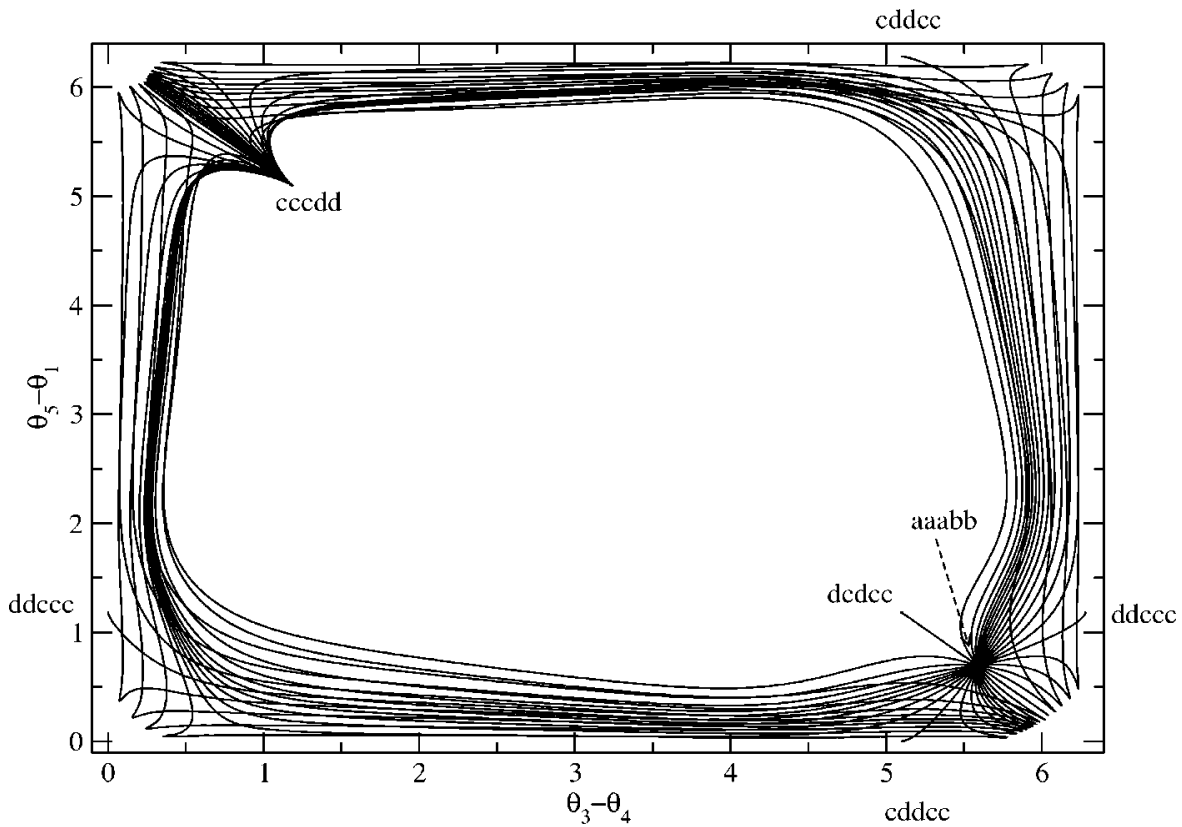

FIG. 3. A large number of trajectories are started on the two dimensional unstable manifold of the periodic orbit $a a a b b$ in the lower right of the figure. Forty different initial conditions are started within $10^{-3}$ of the orbit and the phase differences between oscillators are shown. Observe that almost all trajectories lie within the stable manifold of cccdd; however, there are exceptional trajectories (lying within subspaces with symmetry $\mathbf{S}_{1} \times \mathbf{S}_{2}$ $\times \mathbf{S}_{2}$ ) that converge to $d d c c c$, $d c d c c$, and $c d d c c$. These give rise to switches between conjugate symmetries. periodic orbits and after a while a numerically induced stability sets in, owing to the fact that rounding maps the trajectory into one of the invariant subspaces (see also, for example, Ref. [9]). However, we have no argument that rules out dynamics-induced switching between conjugate attractors in the noise-free system.

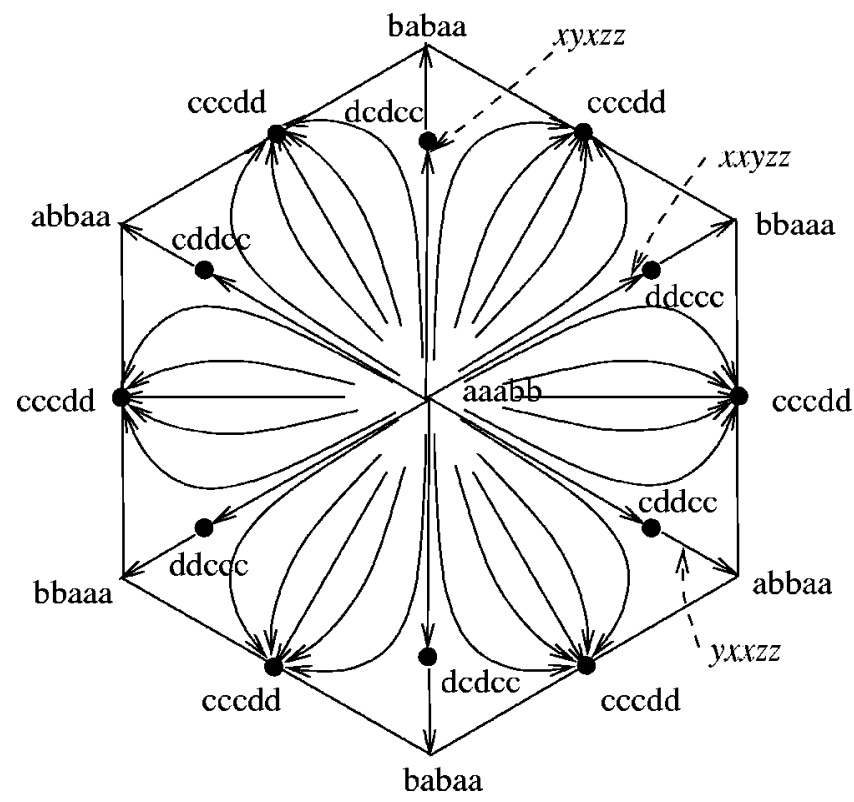

FIG. 4. Schematic diagram showing the geometry of the unstable manifold of the periodic orbit aaabb modulo the group orbit by $\mathbf{T}$. Note that almost all trajectories from $a a a b b$ are asymptotic to cccdd. However, the exceptional trajectories that are in the $\mathbf{S}_{1}$ $\times \mathbf{S}_{2} \times \mathbf{S}_{2}$ symmetry subspaces $x y x z z, x x y z z$, and $y x x z z$ converge to different equilibria. Trajectories from $a a a b b$ to $c c c d d$ that pass for example near $d d c c c$ will also pass near bbaaa and so the periodic orbits bbaaa, babaa, and abbaa are also contained within the closure of the unstable manifold of $a a a b b$. The accessible conjugate periodic orbits to $c c c d d$ are shown as black dots.
In the presence of isotropic noise as in Fig. 1 one can readily identify the presence of occasional switches in symmetry and numerical evidence indicates that as the noise $\eta$ $\rightarrow 0$ the probability of transitions between different symmetry types goes to zero with amplitude $\eta>0$. We can explain this in terms of picturing the heteroclinic network in the presence of noise as an attractor that is roughly speaking an $\eta$ neighborhood of the noise-free network. This means that within $O(\eta)$ of the "exceptional" connections, for example, near $a b b a a$ on the unstable manifold of $a a a b b$, there is a high chance that trajectories will be taken into a neighborhood of the subspace $x y y z w$ containing abbaa. In this way, a trajectory in the presence of noise can "tunnel" its way around the network shown in Fig. 5.

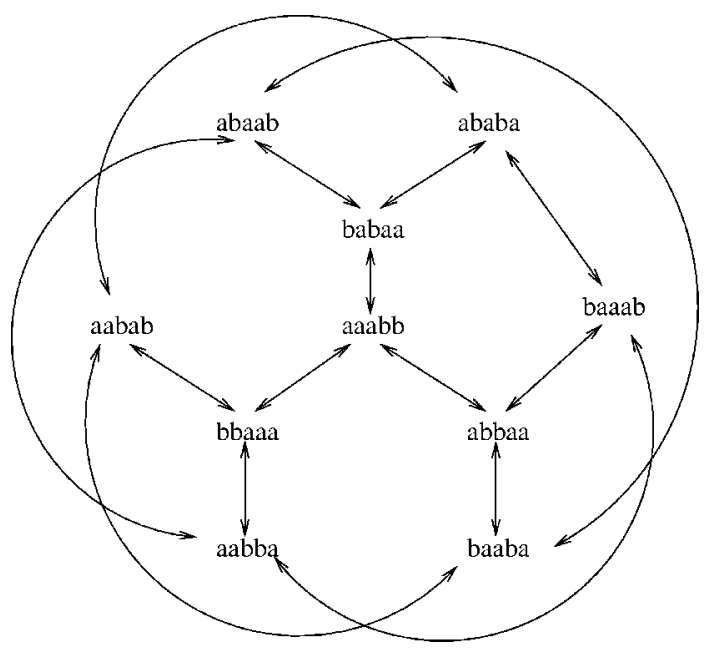

FIG. 5. This diagram shows the structure of connections between conjugate symmetries of the form $\mathbf{S}_{2} \times \mathbf{S}_{3}$. The arrows indicate the transitions that are possible by choosing a heteroclinic trajectory in the network. Observe that starting at any state, for example $a a a b b$, three of the nine conjugate states are accessible after one step; the remaining six are accessible after two steps. 


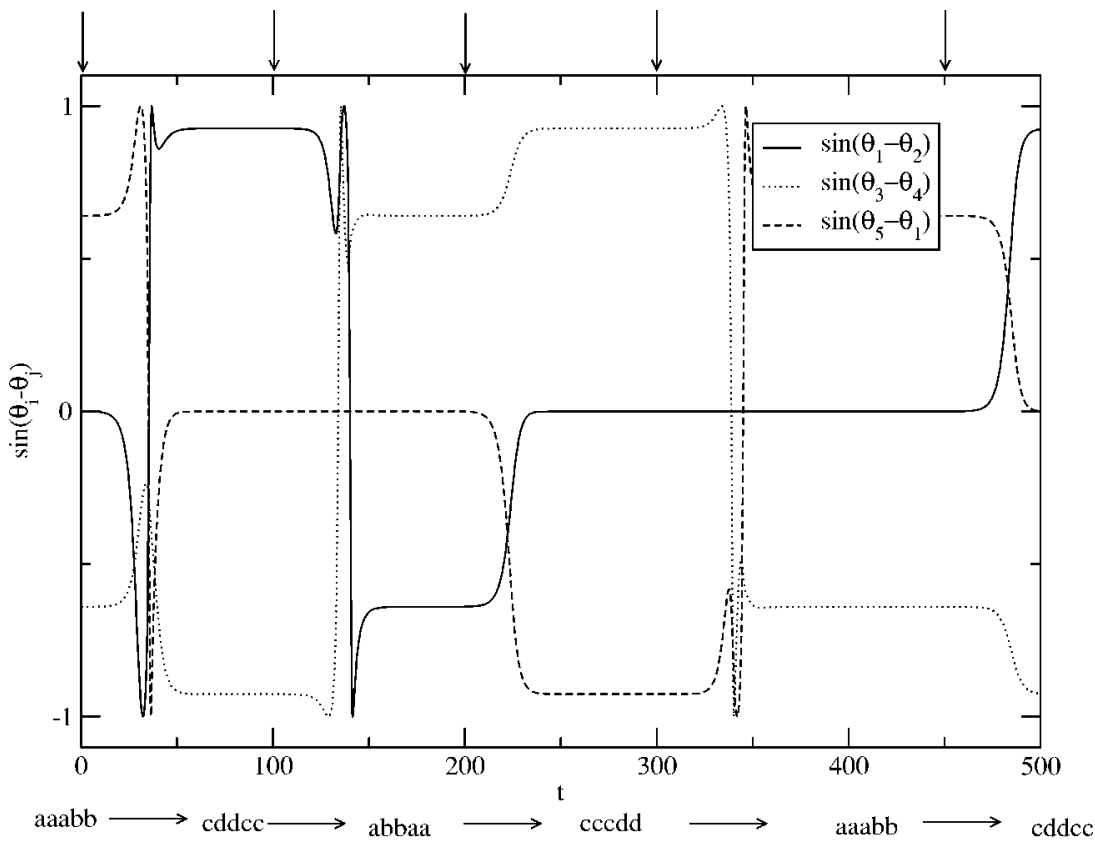

FIG. 6. An illustration of perturbation-induced control of the transitions between $\mathbf{S}_{2} \times \mathbf{S}_{3}$ periodic orbits, by impulsively applying perturbations of order $10^{-5}$ in the directions of chosen symmetry subspaces, at the times indicated by the arrows above the graph. This suggests that (a) the network is highly efficient at encoding a state as a particular conjugate symmetry, (b) the chosen switching between symmetries is obtained on a fast time scale by small perturbations of the system and (c) the network is only sensitive to perturbations at certain points in time, namely those where it is near a periodic orbit conjugate to one of type $a a a b b$.

To illustrate the controllable characteristics of this network, we show in Fig. 6 an example, in the absence of noise, of how one can traverse the network using only very small perturbations. Starting on the periodic orbit $a a a b b$ we apply perturbations of order $10^{-5}$ at the time locations indicated by the arrows above the plot. The perturbations are chosen to be within the unstable manifolds of each of the periodic orbits. For example, if we perturb

$$
(a, a, a, b, b) \mapsto(a+x, a, a, b, b)
$$

for $x$ very small we find that the system proceeds to the periodic orbit $(c, d, d, c, c)$. Figure 6 shows a series of transitions

$$
a a a b b \mapsto c d d c c \mapsto a b b a a \mapsto c c c d d \mapsto a a a b b \mapsto c d d c c \cdots
$$

In principle one can explore any path on the directed graph Fig. 5 using this method.

\section{SWITCHING BETWEEN CONJUGATE SYMMETRIES IN A PHASE-ENERGY MODEL}

We consider a simple phase-energy model that is a natural extension of Refs. [9] and [10] while yielding additional complexity in that the oscillators are two dimensional. These phase-energy equations (introduced by Ref. [22]) are posed on the space $\mathbf{T}^{n} \times \mathbf{R}^{n}$ and have the form

$$
\dot{\theta}_{i}=\omega\left(\alpha_{i}\right)
$$

$$
\dot{\alpha}_{i}=-\beta\left(\alpha_{i}\right)+\frac{\epsilon}{N} \sum_{i=1}^{N} g\left(\theta_{i}-\theta_{j}\right),
$$

where the phase of the oscillator $i$ is represented by the angular variable $\theta$ (modulo $2 \pi$ ) and the energy relative to the uncoupled system $(\epsilon=0)$ is represented by $\alpha_{i} \in \mathbf{R}$. Note that $\beta>0$ is necessary for the term $-\beta(\alpha)$ in Eq. (3) to stabilize
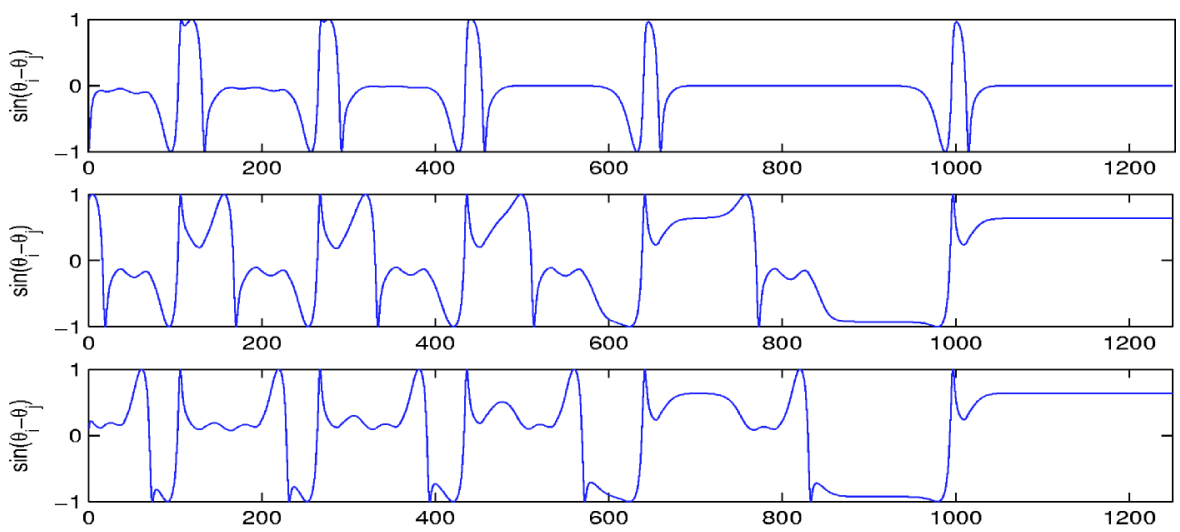

FIG. 7. Time series plot of the sines of the differences between the phases for a system of five coupled phase-energy oscillators $\lambda_{1}=-0.4, \lambda_{2}=0, \beta=1, \epsilon=1, \delta$ $=4.391, r=0.25$.

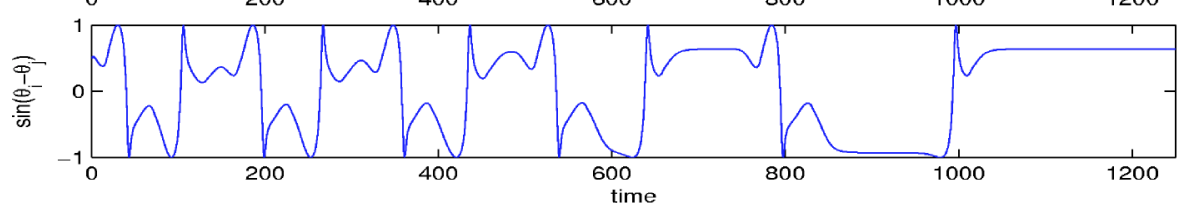



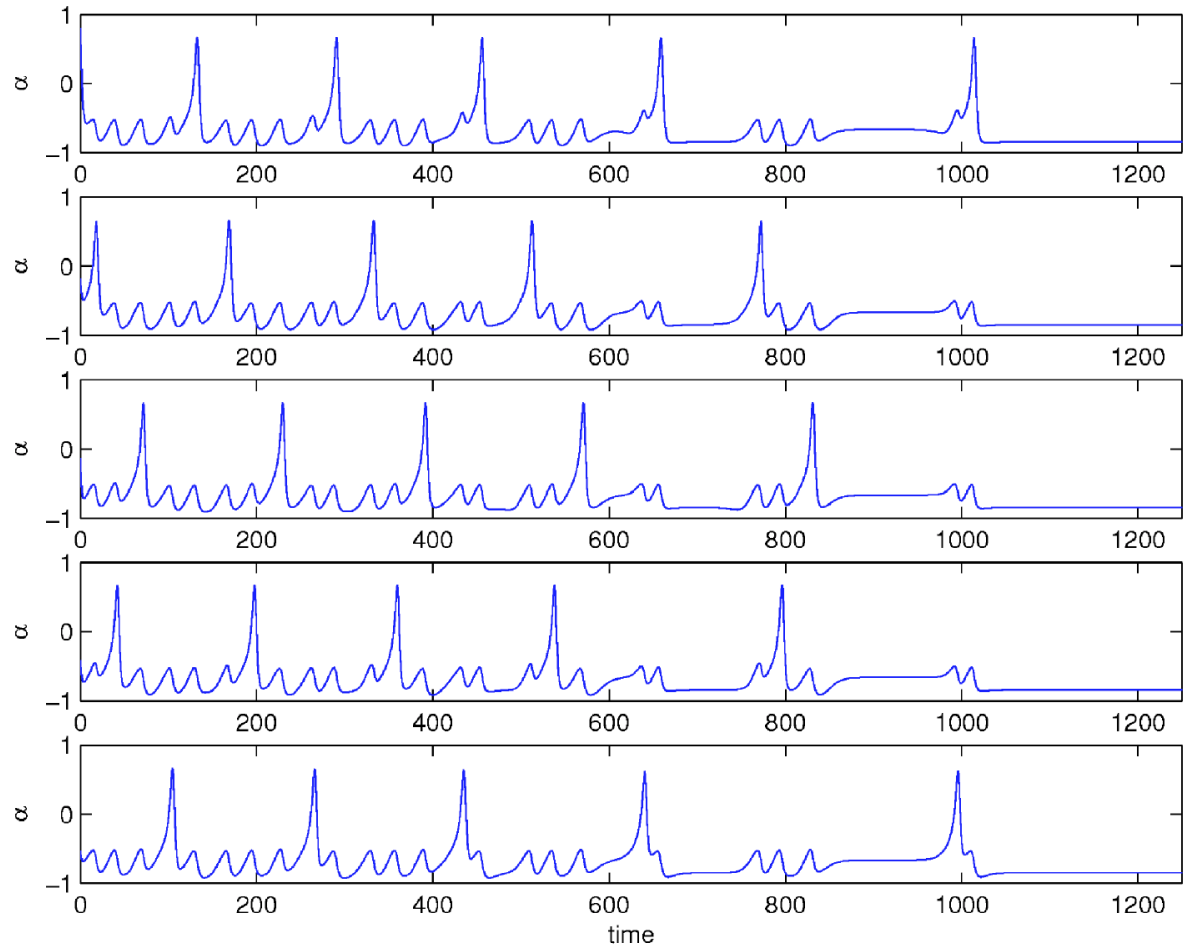

FIG. 8. Time series plot of the sines of the amplitudes for a system of five coupled phase-energy oscillators $\lambda_{1}=-0.4, \lambda_{2}=0, \beta$ $=1, \epsilon=1, \delta=4.391, r=0.25$. the oscillations. The model can be motivated by noting that it gives the most significant terms for weakly dissipative coupled Hamiltonian oscillators. We set

$$
\omega(\alpha)=1+\lambda_{1} \alpha+\lambda_{2} \alpha^{2}
$$

By choosing the function $g(\phi)$ in Eq. (3) above to be Eq. (2), the interactions present in coupled neural systems correspond to those in the Hansel et al. model. On simulating the model (2)-(4) we obtain very similar time series as those for the phase oscillator; examples are plotted in Figs. 7 and 8. In Fig. 7 periods of synchronization between oscillators 1 and 2 are clearly apparent, however do not persist throughout the integration. Again, slow oscillations between clustered periodic states of symmetry $\mathbf{S}_{2} \times \mathbf{S}_{3}$ are apparent.
The dynamics of the clusters of heteroclinic cycles were found using the Leader algorithm as in Ref. [23]. Figure 9 shows the behavior of clusters in the presence of small amplitude noise. After transients, the dynamics settle to a cycle moving between the stable $(3,2)$ cluster states (represented in Fig. 9 by the periods where two clusters are present) with two distinct trajectories between them. The first trajectory exhibits only a single increase in the number of clusters before returning to a two cluster state, the second a longer trajectory where the clusters break until four clusters are detected (only two of the oscillators are now synchronized).

Finally, Fig. 10 shows a longer time series from the coupled phase-energy oscillators (2)-(4) with the addition of noise of order $10^{-7}$. One can observe a number of noiseinduced switches between slow oscillations with conjugate

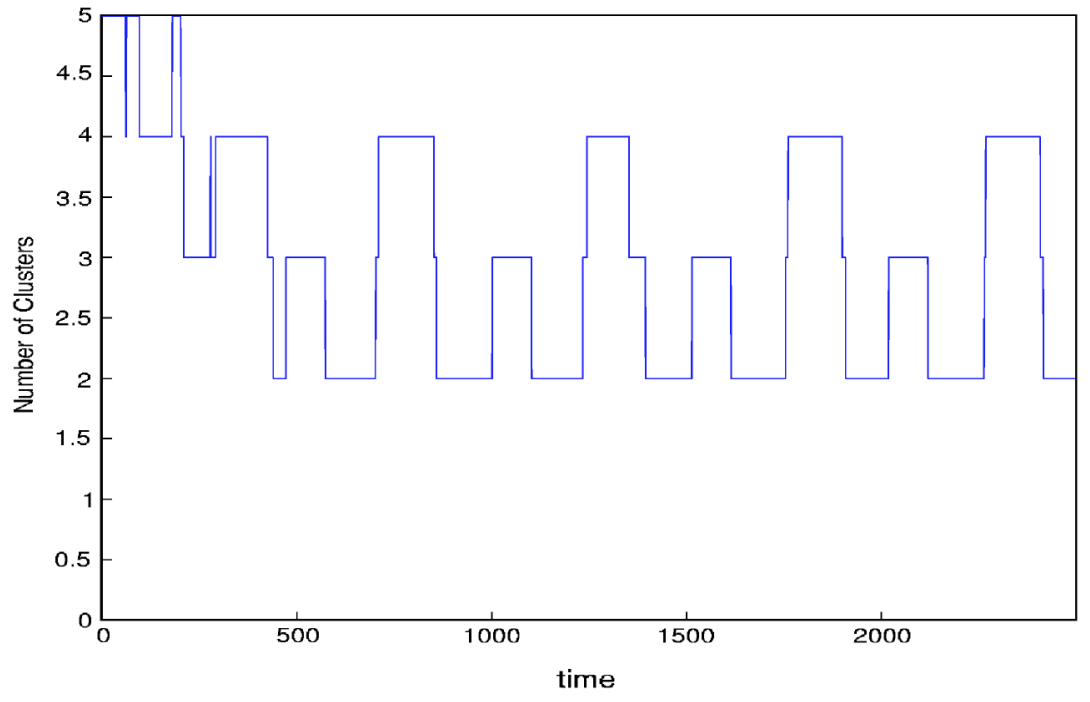

FIG. 9. Time series plot of the number of clusters (tolerance $10^{-4}$ ) for a system of five coupled phase-energy oscillators as in Figs. 7 and 8 in the presence of noise with amplitude $10^{-10}$. Observe that the repeated clustering and cluster breaking follows two distinct cycles between states where two clusters are present. The switching between conjugate symmetries is only possible via small perturbations if they are applied just before the cluster of two splits into four clusters. 

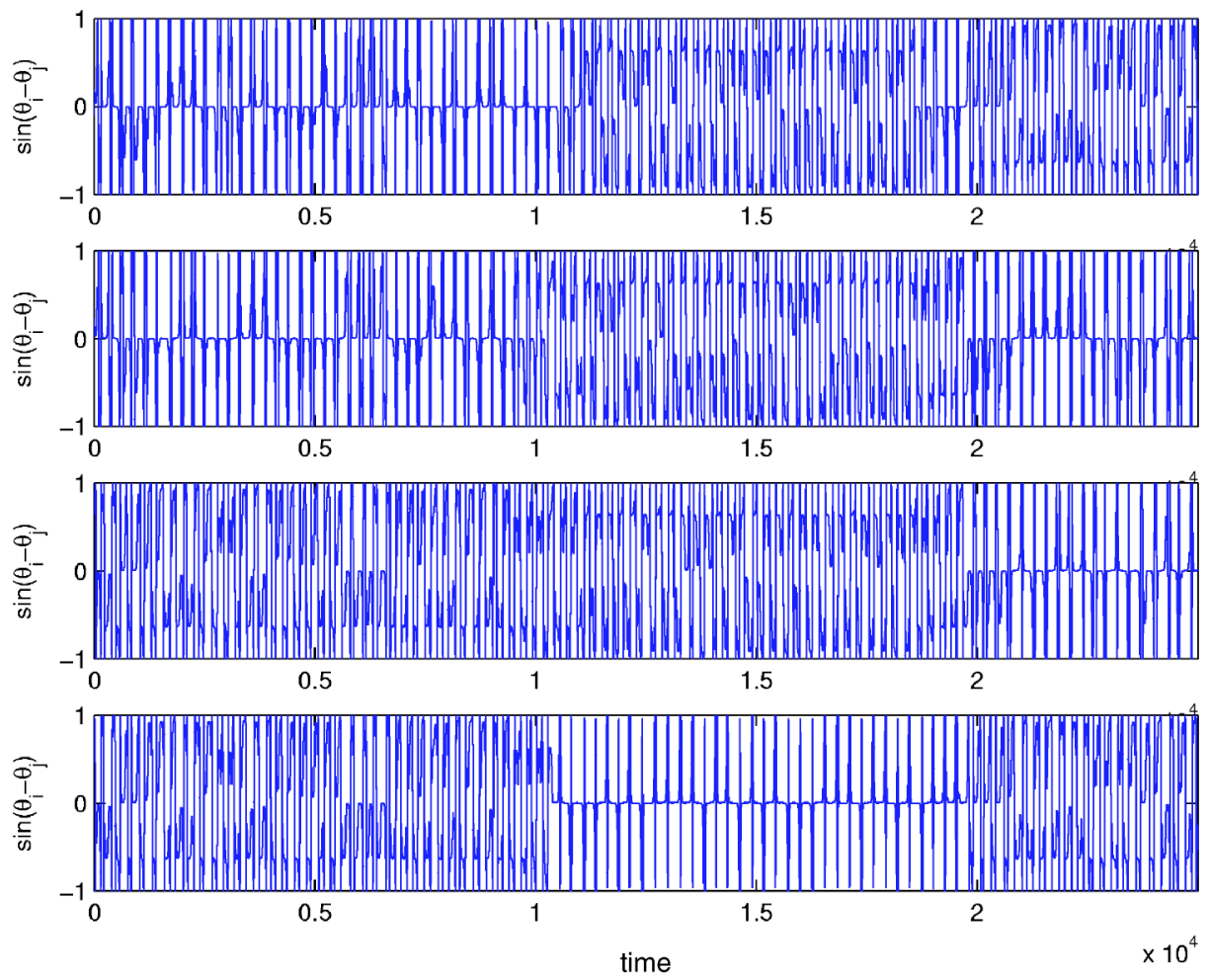

FIG. 10. Time series of five coupled oscillators, noise amplitude $10^{-7}$. Observe that several switches occur during the time series, as demonstrated by phases during which oscillators are approximately synchronized into the cluster states. The slow oscillations between cluster states occur on an average of period 300 while the switching between conjugate symmetries occurs after an average time of order $10^{4}$.

symmetries; most noticably at times $t=1.05 \times 10^{4}$ and at $t$ $=1.95 \times 10^{4}$. Thus we find equivalent dynamical behavior to the simpler phase oscillators model (1) in a model with twodimensional oscillators.

\section{DISCUSSION: ENCODING USING CONJUGATE SYMMETRIES}

We have examined the heteroclinic attractor of Ref. [9] for five globally coupled phase oscillators. This consists of slow oscillations between periodic states with symmetry $\mathbf{S}_{2} \times \mathbf{S}_{3}$. In the presence of small perturbations, we have shown examples where switching within the network occurs.

The dynamics of this network suggests that this is a good mechanism for modeling the reversible encoding of a high number of "memory states" within a highly coupled network. In particular:

(i) The attractor is robust to noise and perturbations; these just affect the rate of slow oscillations.

(ii) It is easy to robustly direct the network into any of a number of conjugate states.

(iii) There is a natural "clocking rate" associated with network, determined by the level of noise forcing. Switching between conjugate attractors is only possible at distinct times where one passes near solutions of type $a a a b b$.

There are a large number of conjugate cluster states (this will grow exponentially with number of oscillators) and these are arranged in a network with short diameter; one can go between any two of the ten conjugate $\mathbf{S}_{2} \times \mathbf{S}_{3}$ symmetric states using at most two switches. Moreover, we observe that a switch between slow oscillations with conjugate symmetries is easy to arrange simply by providing a small perturbation to one of the oscillators.

All of these properties are highly desirable for encoding of memory states, and it is a matter for future research to scale these results up to higher dimensional systems; in [24] we illustrate how one can use the heteroclinic network discussed in this paper as a unit for memory and timing within a computational network. We end by emphasizing that the order parameter $\chi$ cannot be used to illustrate the effects of switching between conjugate symmetries simply because it is invariant under the symmetry group.

\section{ACKNOWLEDGMENTS}

We gratefully acknowledge discussions with Marc Timme and Gerhard Dangelmayr concerning this research. The research of J.B. was supported by EPSRC.
[1] S. C. Manrubia and A. S. Mikhailov, Europhys. Lett. 53, 451 (2001).

[2] M. Breakspear, Int. J. Neural Syst. 11, 101 (2001).

[3] K. Kaneko, Physica D 75, 53 (1994).
[4] Y. Q. Wang and Z. D. Wang, Phys. Rev. E 62, 1063 (2000).

[5] B. Bente, G. Giovanni, R. Brandt, and H. Mustaparta, J. Comp. Neurol. 446, 123 (2002).

[6] S. H. Strogatz, Nature (London) 410, 268 (2001). 
[7] O. Popovych, A. Pikovsky, and Y. Maistrenko, Physica D 168-169, 106 (2002).

[8] Y. Maistrenko, O. Popovych, and M. Hasler, Int. J. Bifurcation Chaos Appl. Sci. Eng. 10, 179 (2000).

[9] D. Hansel, G. Mato, and C. Meunier, Phys. Rev. E 48, 3470 (1993).

[10] H. Kori and Y. Kuramoto, Phys. Rev. E 63, 046214 (2001).

[11] H. Kori, Phys. Rev. E 68, 021919 (2003).

[12] M. Timme, F. Wolf, and T. Geisel, Phys. Rev. Lett. 89, 154105 (2002).

[13] W. Gerstner and K. Kistler, Spiking Neuron Models (Cambridge University Press, Cambridge, England, 2002).

[14] J. L. Hindmarsh and R. M. Rose, Proc. R. Soc. London, Ser. B 221, 87 (1984).

[15] F. C. Hoppensteadt and E. Izhikevich, Weakly Connected Neural Networks (Springer, New York, 2000).
[16] P. Ashwin and J. W. Swift, J. Nonlinear Sci. 2, 69 (1992).

[17] D. Hansel, G. Mato, and C. Meunier, Europhys. Lett. 23, 367 (1993).

[18] M. Rabinovich, A. Volkovskii, P. Lecanda, R. Huerta, H. D. Abarbanel, and G. Laurent, Phys. Rev. Lett. 87, 068102 (2001).

[19] P. Ashwin and G. Dangelmayr (unpublished).

[20] K. Kaneko, Physica D 41, 137 (1990).

[21] A. S. Pikovsky, M. G. Rosenblum, and J. Kurths, Europhys. Lett. 34, 165 (1996).

[22] P. Ashwin and G. Dangelmayr, Dyn. Stab. Syst. 15, 263 (2000).

[23] J. A. Hartigan, Clustering Algorithms (Wiley, New York, 1975).

[24] P. Ashwin and J. Borresen (unpublished). 